\title{
Self-Tuning Synthesis Filter against Mutual Coupling and Interferences for GNSS and Its Implementation on Embedded Board
}

\author{
Chung-Liang Chang \\ Department of Biomechatronics Engineering, National Pingtung University of Science and Technology, Pingtung 91201, Taiwan
}

Correspondence should be addressed to Chung-Liang Chang, chungliang@mail.npust.edu.tw

Received 10 January 2010; Revised 2 May 2010; Accepted 9 June 2010

Academic Editor: George Tombras

Copyright (C) 2010 Chung-Liang Chang. This is an open access article distributed under the Creative Commons Attribution License, which permits unrestricted use, distribution, and reproduction in any medium, provided the original work is properly cited.

Traditional spatial-temporal adaptive signal processing techniques are often applied to conduct narrowband and wideband interferences. However, its mitigation performance degrades greatly due to mutual coupling. To solve this problem, this paper aims to utilize a spatial-temporal self-tuning synthesis filter capable of mutual coupling compensation and interference mitigation. The spatial filter and temporal filter are to compensate for the effect of mutual coupling and interference mitigation, respectively. Self-tuning mechanism is to adopt least square (LS) and minimum variable distortionless response- (MVDR-) based method to adjust spatial and temporal weights of antenna array. The experiment platform is established by the embedded development board. Simulation and experiment results demonstrate that the proposed method can effectively compensate for mutual coupling, mitigate the cochannel interference up to $30 \mathrm{~dB}$, and enhance the acquisition performance of receivers in global navigation satellite system (GNSS).

\section{Introduction}

Satellites of global positioning system (GPS) operate on the orbit 20200 kilometers high and the power of carrier for L1 band upon the ground is about $-130 \mathrm{dBm}$. In general, the power of thermal noise can reach $-110 \mathrm{dBm}$. It indicates that the power of GPS signal is lower than that of thermal noise. Luckily, GPS adopts spread spectrum technique to acquire sufficient gain for reception end to reconstruct signals. However, if GPS is susceptible to unintentional or intentional interference (jammer), it results in the difficulty in reception and lock of GPS signals due to over strong magnitude of interference, too wide bandwidth, and similarity in type of modulation.

Typical interference mitigation techniques consist of three types. The first is defined as antenna array model, which utilizes multiple antennas to conduct the estimation of interference direction and adjust the gain and phase of antenna sets to null out interferences. The second is termed precorrelation processing, which transfers high-frequency to intermediate-frequency (IF) analog or digital signal and then conducts signal processing regarding amplitude, time domain, or frequency domain to decrease the impact of interference $[1,2]$. The last is called postcorrelation processing, which adopts software-defined radio to integrate GPS with other assisted navigation devices to enhance the precision in navigation positioning. With regards to the effect of interference mitigation, antenna array model is most efficient. The precorrelation processing technique is medium in effect and cost, but the reception circuit has to be redesigned. The third technique is limited in effect though it can employ available receivers in global navigation satellite system (GNSS) and additional inertial navigation components to aid positioning.

In a word, in terms of interference mitigation, the immediate removal or mitigation of interferences upon the radio frequency (RF) front-end of receiver yields a better result than that upon rear-end of the receiver. Due to the specific of geometric relations among each antenna component, associated phase relation exists among received signals. The use of spatial signal processing techniques can determine the direction of interference source, and the adoption of adaptive 
algorithm can establish spatial filter to eliminate the impact of interference in GNSS receiver [3]. The insertion of time domain filter to spatial signal processing can be extended to spatial-temporal adaptive signal processing [4-6], which performs by selecting the adaptive weights in order to maintain the desired GPS signal and meanwhile minimize all interferences. The key in spatial-temporal signal processing is the use of adaptive algorithm to obtain the optimal weight, which is multiplied with received signal of each antenna and then summed altogether to yield the minimal error between array output and design signal [7]. The constructed adaptive beamforming is established through desired steering vector without mutual coupling. Provid such ideal condition as the equal spacing between antenna (half wave length) and no consideration for antenna characteristics, and so forth, many factors in reality generate mutual coupling, such as the spacing between antenna less than half wavelength, thermal noise, or the variation in radiation parameter and nonideal characteristics in the design of antenna itself under highfrequency environment.

Earlier, Sarkar and Sangruji propose direct data domain approach, which adaptively minimizes the interference power and maintains array gain in the direction of the signal [8], even if this method can cancel out undesired interference sources. However, the effect of mutual coupling on beamforming results in the error in direction of arrival (DoA). As a result, it causes error of steering vector, failure to mitigate interference due to wrong DoA and strengthen the direction gain of desired signal. Besides, the direction gain does not null further.

An unfavorable impact of mutual coupling on the performance of adaptive array algorithms has been reported $[9,10]$. Friel and Pasala pointed out that the mutual coupling greatly affects the signal-to-interference plus noise ratio (SINR) of array output [10]. In 2000, Adve et al. utilized method of moments (MOMs) as well as direct data domain technique [9] to access mutual coupling between the elements of a given array.

In order to reduce the adverse effects of mutual coupling on antenna arrays, several mathematical models of mutual coupling have been proposed in the literature review over the last two decades [11-15]. In 1999, Svantesson estimates the mutual coupling using electromagnetic concepts. Then, the directions and coupling parameters are estimated utilizing maximum likelihood method. However, it is not possible neither to cancel out its effect nor to predict its variation as the electromagnetic environment adjacent the antenna changes. The autocalibration algorithm was employed to compensate for mutual coupling in uniform and linear arrays [13]. Both the DoAs of the incoming signals and the unknown mutual coupling matrix of the array were estimated in this algorithm.

The narrowband applications and proper compensation of the induced surface current mutual coupling matrix $(\mathrm{MCM})$ can be estimated off-line $[10,14,16]$. The online estimation should be utilized when the electromagnetic environment that surrounds the antenna fails to be appropriately compensated and the antenna operates with wideband signal [13]. However, MCM is estimated either on-line or off-line, whose structure is unchangeable. That is, it is symmetric, per-symmetric, and Toeplitz. Suppose MCM is known; mutual coupling can be mitigated to a large scale through multiplication with the inverse of the coupling matrix (suppose it to be full rank) [12]. Note that in a large regular array, the edge effects may be ignored and the mutual coupling matrix reduces to an identity matrix except for a scaling factor. The above depiction shows that $\mathrm{MC}$ compensation is operated in two steps.

The first step is to accurately estimate MCM. The second step is the act of compensation, which is to premultiply the data vector ot transmission weights by inverting MCM. Thus, MC can be compensated utilizing the compensation processor before the input signal is processed by adaptive beamformer or DoA processor or after computation of the transmission weights.

In this paper, we assume the MCM known a priori; the proposed MC compensator utilizes least square (LS) method depending on iteration process. Then, the estimated mutual coupling matrix is adopted to reduce the effect on interference mitigation. In addition, minimum variance distortion less response- (MVDR-) based temporal processing is employed to cancel wideband interference and aid $Q R$-decomposed operator to shorten computation time. The novelty of this paper is that it is not only capable of mitigating narrowband and wideband interferences but also capable of providing a solution for mutual coupling compensation. Besides, the proposed method is in reality verified on Altera-embedded development board to evaluate the effect of mutual coupling compensation and interference mitigation.

The remainder of the paper is organized as follows. Section 2 describes the application of proposed method to mutual coupling and interference. The simulation results are demonstrated in Section 3. This is followed by a description of the development of embedded system in Section 4 . The experimental results of the antenna array against mutual coupling and pseudolite-type interferences are also given in this section. Finally, some conclusions are drawn in Section 5 .

\section{Methodology}

2.1. Signal Array Model. This section presents the mathematical model of array output signal when the array signal is influenced by mutual coupling. Firstly, the mathematical model unsusceptible to mutual coupling is analyzed, which has been completed [7]. The following then depicts the mathematical model under the influence of mutual coupling and illustrates the mutual coupling matrix model.

Assume that the real received baseband signal at time instant $k$ of $N$ antenna elements with $M$-tap temporal filter for each antenna is described by an $N(M+1) \times 1$ vector shown as follows:

$$
\begin{aligned}
\mathbf{x}(k) & =\mathbf{x}_{s}(k)+\mathbf{x}_{J}(k) \\
& =\underbrace{\sqrt{P_{s}} \mathbf{A s}(k)}_{\mathbf{x}_{s}(k)}+\sum_{j=1}^{J} \sqrt{P_{j}} \mathbf{B}_{j} \mathbf{i}_{j}(k)+\mathbf{C}(k),
\end{aligned}
$$


where $\mathbf{x}(k)=\left[\begin{array}{lll}\mathbf{x}_{\mathrm{a}}(k) & \mathbf{x}_{\mathrm{b}} & (k)\end{array}\right]^{\mathrm{H}}$ depicts the incoming signal samples on the taps of spatial-temporal filter. $[\cdot]^{\mathrm{H}}$ denotes the Hermitian transpose operator. $\mathbf{x}_{\mathrm{a}}(k)=\left[x_{1}^{\mathrm{a}}(k) \cdots\right.$ $\left.x_{N}^{\mathrm{a}}(k)\right]$ and $\mathbf{x}_{\mathrm{b}}(k)=\left[\begin{array}{llll}\mathbf{x}_{1}^{\mathrm{b}}(k) & \mathbf{x}_{2}^{\mathrm{b}}(k) & \cdots & \mathbf{x}_{M}^{\mathrm{b}}(k)\end{array}\right]$ are the spatial and temporal signal snapshots, respectively. $\mathbf{x}_{m}^{\mathrm{b}}(k)=\left[x_{1 m}(k)\right.$ $\left.\cdots x_{N m}(k)\right]$ and $\mathbf{s}(k)=\left[\begin{array}{llll}s_{1}(k) s_{2}(k) & \cdots & s_{M+1}(k)\end{array}\right]^{\mathrm{H}}$ are both desired signals and essential modulated spread codes subject to data modulation, time delay, Doppler shift, and phase variation that uncorrelated with the channel noise vector. $P_{s}$ and $P_{j}$ are the power of desired signal and $j$ th interference, respectively. $\mathbf{i}_{j}(k)$ has the same structure as $\mathbf{s}(\mathrm{k})$, and $J$ is the total number of interferences. Each interference can be modeled as narrowband or wideband signal. $\mathbf{C}(k)$ is zero-mean, temporally and spatially white noise with variance $\sigma^{2} \mathbf{I}$. A $=\mathbf{I}_{(M+1) \times(M+1)} \otimes \mathbf{a}_{\theta_{s}}, \phi_{s}$ and $\mathbf{B}_{j}=\mathbf{I}_{(M+1) \times(M+1)} \otimes \mathbf{a}_{\theta_{j}, \phi_{j}}$, where $\otimes$ denotes the Kronecker product. $\mathbf{a}_{\theta_{s}, \phi_{s}}=\left[\begin{array}{llll}a_{1}^{s}(k) & a_{2}^{s}(k) & \cdots & a_{N}^{s}(k)\end{array}\right]^{\mathrm{H}}$ and $\mathbf{a}_{\theta_{j}, \phi_{j}}=\left[\begin{array}{llll}a_{1}^{j}(k) & a_{2}^{j}(k) & \cdots & a_{N}^{j}(k)\end{array}\right]^{\mathrm{H}}$ denote the $N \times 1$ steering vector with respect to desired satellite and $j$ th interference source, respectively.

The array vector model depicted by (1) is valid only for ideal arrays. For practical arrays, the simultaneous presence of more than one sensor as well as objects adjacent to the array accounts for the noted mutual coupling effect. In a more realistic scenario, the signal received by one sensor can be expressed as a linear combination of the wave fields incidents onto all the sensors rather than associated with the wave field incident on that sensor only. To take the coupling effect into consideration, the array signal model in (1) has to be modified by incorporating a matrix term called coupling matrix. Therefore, the $\mathbf{A}$ and $\mathbf{B}_{j}$ in the presence of mutual coupling can be written as

$$
\begin{aligned}
\overline{\mathbf{A}} & =\mathbf{I}_{(M+1) \times(M+1)} \otimes \mathbf{M a}_{\theta_{s}, \phi_{s}}, \\
\overline{\mathbf{B}}_{j} & =\mathbf{I}_{(M+1) \times(M+1)} \otimes \mathbf{M a}_{\theta_{j}, \phi_{j}},
\end{aligned}
$$

where $\mathbf{M}$ is an $N \times N$ matrix that demonstrates the mutual coupling effect, introduces the distortion of amplitudes and phases in elements of steering matrix, and depicts how the individual antenna elements are coupled with one another. Nevertheless, mutual coupling coefficients between two far apart elements can be approximated to zero. Thus, it is adequate to consider the coupling model with just a few nonzero coefficients. The mutual coupling matrix (MCM) can be given as follows:

$$
[\mathbf{M}]_{p q}= \begin{cases}\delta, & |p-q|=1 \\ 1, & p=q \\ 0, & \text { other }\end{cases}
$$

where []$_{p q}$ indicates the coupling contribution of the $q$ th antenna to $p$ th antenna and $\delta$ is the intensity of the received power of its immediate neighbors.
Thus, the array signal vector in the presence of mutual coupling can be rewritten as

$$
\widetilde{\mathbf{x}}(k)=\sqrt{P_{s}} \overline{\mathbf{A}} \mathbf{s}(k)+\sum_{j=1}^{J} \sqrt{P_{j}} \overline{\mathbf{B}}_{j} \mathbf{i}_{j}(k)+\mathbf{C}(k),
$$

where $\tilde{\mathbf{x}}=\left[\tilde{\mathbf{x}}_{\mathrm{a}}(k) \tilde{\mathbf{x}}_{\mathrm{b}}(k)\right]^{\mathrm{H}}$ is the same structure as (1). Finally, it is possible to write the spatial covariance matrix of the array signal vector in the absence and presence of mutual coupling as

$$
\begin{aligned}
& \mathbf{R}_{11} \triangleq E\left\{\mathbf{x}_{1}^{\mathrm{H}}(k) \mathbf{x}_{1}(k)\right\}, \\
& \widetilde{\mathbf{R}}_{11} \triangleq E\left\{\widetilde{\mathbf{x}}_{1}^{\mathrm{H}}(k) \tilde{\mathbf{x}}_{1}(k)\right\},
\end{aligned}
$$

where $\tilde{\mathbf{x}}_{m}(k)=\left[\begin{array}{lll}\tilde{x}_{1 m}(k) & \cdots & \tilde{x}_{N m}(k)\end{array}\right]$ and $E\{\cdot\}$ denote the expectation operator. Similarly, the temporal covariance matrix of the array signal vector in the absence and presence of mutual coupling is shown as follows:

$$
\begin{aligned}
& \mathbf{R}_{\mathrm{bb}} \triangleq E\left\{\mathbf{x}_{\mathrm{b}}^{\mathrm{H}}(k) \mathbf{x}_{\mathrm{b}}(k)\right\}, \\
& \widetilde{\mathbf{R}}_{\mathrm{bb}} \triangleq E\left\{\tilde{\mathbf{x}}_{\mathrm{b}}^{\mathrm{H}}(k) \tilde{\mathbf{x}}_{\mathrm{b}}(k)\right\} .
\end{aligned}
$$

2.2. Mutual Coupling Compensation and Interferences Mitigation. The rules in the design of adaptive antenna algorithm can be applied in spatial and temporal domain. The structure of an adaptive antenna is adapted through spatial filters whose complex weights combine the signal from each antenna element. Each antenna element is processed with its own temporal filter, the outputs of which are joined together to yield a single output signal. The weights are selected to effectively null out interference while intentionally retaining the desired signal. The adaptive nulling of the antenna array is demonstrated by the capability to steer several nulls (minimum of the radiation pattern) to the interferences while keeping a maximum of the radiation pattern toward the desired signal. However, the above process of adaptive array algorithm is undertaken without considering mutual coupling effect. To evaluate the effect of mutual coupling and compensate for its influence, an adaptive spatial filter with LS method is utilized to make up for mutual coupling effect and a temporal filter with $Q R$ based MVDR beamformer is adopted to mitigate wideband and narrowband interferences.

2.2.1. Mutual Coupling Compensation. It is shown from (2) that when MCM is known, mutual coupling effect can be easily nulled out by premultiplying the input steering vector $\mathbf{a}_{\theta, \phi}$ by the inverse of MCM (assuming that it is invertible). Assume that the weight vector $\mathbf{w}_{\mathrm{a}}$ is susceptible to mutual coupling; the estimated one is expressed by

$$
\tilde{\mathbf{w}}_{\mathrm{a}}=\mathbf{M} \mathbf{w}_{\mathrm{a}}+\mathbf{e},
$$

where e denotes the measurement error. The mutual coupling $\mathbf{M}$ can be calculated by employing LS method as a solution for the following optimization problem:

$$
\widetilde{\mathbf{M}}=\arg \min _{\mathbf{M}}\left\|\widetilde{\mathbf{w}}_{\mathrm{a}}-\mathbf{M w}_{\mathrm{a}}\right\|_{2}^{2},
$$


where $\|\cdot\|_{2}$ indicates the Euclidean norm, and $\mathbf{w}_{\mathrm{a}}=\mathbf{a}_{\theta_{s}, \phi_{s}}$ denotes the receiver weight vector for nonadaptive array, or $\mathbf{w}_{\mathrm{a}}=\mathbf{R}_{11}^{-1} \mathbf{a}_{\theta_{s}}, \phi_{s}$ means the receiver optimum weight vector for adaptive array. Thus, $\widetilde{\mathbf{M}}$ can be calculated through LS method:

$$
\widetilde{\mathbf{M}}=\widetilde{\mathbf{w}}_{\mathrm{a}} \mathbf{w}_{\mathrm{a}}^{\mathrm{H}}\left(\mathbf{w}_{\mathrm{a}} \mathbf{w}_{\mathrm{a}}^{\mathrm{H}}\right)^{-1} .
$$

2.2.2. MVDR Beamformer. In spatial-temporal processing, the MVDR beamforming can minimize the combined output from an antenna array, in a least square sense limited by independent linear equality constraints (constraint optimization), each of which relates to a selected look direction. Suppose that the constraint is formulated in order to minimize the variance (the average power) of a beamformer output. Meanwhile, distortionless response is maintained in particular direction (target direction of interest). Such a solution is termed MVDR. The cost function is to minimize the output power of array system given by

$$
\begin{array}{ll}
\min _{\mathbf{w}} & \mathbf{w}_{\mathrm{b}}^{\mathrm{H}} E\left[\mathbf{x}_{\mathrm{b}}(k) \mathbf{x}_{\mathrm{b}}^{\mathrm{H}}(k)\right] \mathbf{w}_{\mathrm{b}}=\mathbf{w}_{\mathrm{b}}^{\mathrm{H}} \mathbf{R}_{\mathrm{bb}} \mathbf{w}_{\mathrm{b}} \\
\text { s.t. } & \mathbf{w}_{\mathrm{b}}^{\mathrm{H}} \mathbf{d}_{\theta, \phi}=1,
\end{array}
$$

where $\mathbf{d}_{\theta, \phi}$ is $N M \times 1$ desired spatial-temporal steering vector. Use the Lagrange multiplier operator, and the optimum weight vector is shown as follows:

$$
\mathbf{w}_{\mathrm{MVDR}}=\frac{\mathbf{R}_{\mathrm{bb}}^{-1} \mathbf{d}_{\theta, \phi}}{\mathbf{d}_{\theta, \phi}^{\mathrm{H}} \mathbf{R}_{\mathrm{bb}}^{-1} \mathbf{d}_{\theta, \phi}} .
$$

Many practical applications of MVDR beamformers require online calculation of the weights based on (11). It indicates that the covariance matrix in (6) should be estimated and inverted online. Nevertheless, this process is high in computational cost and it may be hard to estimate the sample covariance matrix in real time if the number of samples $N M$ is large. Besides, the numerical calculation of the weights $\mathbf{w}_{\text {MVDR }}$ utilizing the expression (11) may be very unstable if the sample covariance matrix is ill-conditioned. The use of QR decomposition of the incoming signal matrix can yield a numerically stable and computationally efficient algorithm. This matrix is decomposed as $\mathbf{R}_{\mathrm{bb}}=\mathbf{Q R}$, where $\mathbf{Q}$ denotes the unitary matrix and $\mathbf{R}$ indicates the upper triangular matrix. Thus, the QR-based algorithm for calculation of beamformer weights consists of the following three stages.

Step 1. The linear equation system $\mathbf{R}^{\mathrm{H}} \mathbf{u}_{1}=\mathbf{d}_{\theta, \phi}$ is solved for $\mathbf{u}_{1}$, and the solution is $\overline{\mathbf{u}}_{1}^{\mathrm{T}}=\left(\mathbf{R}^{\mathrm{H}}\right)^{-1} \mathbf{d}_{\theta, \phi}$.

Step 2. The linear equation system $\mathbf{R}^{\mathrm{H}} \mathbf{u}_{2}=\overline{\mathbf{u}}_{1}^{\mathrm{T}}$ is solved for $\mathbf{u}_{2}$, and the solution is $\overline{\mathbf{u}}_{2}^{\mathrm{T}}=\mathbf{R}^{-1} \overline{\mathbf{u}}_{1}^{\mathrm{T}}$.

Step 3. The weight vector is obtained as $\widetilde{\mathbf{w}}_{\mathrm{MVDR}}=\overline{\mathbf{u}}_{2}^{\mathrm{T}} / \mathbf{d}_{\theta, \phi}^{\mathrm{H}} \overline{\mathbf{u}}_{2}^{\mathrm{T}}$.

The Matlab's QR decomposition function is utilized in this paper.
2.3. Self-Tuning Synthesis Algorithm. To compensate for mutual coupling effect and mitigate interferences, methods described in Sections 2.2.1 and 2.2.2 are combined to simultaneously solve these problems. Assume that the output of the array system can be illustrated in the following:

$$
\begin{aligned}
y(k) & =\mathbf{w}^{\mathrm{H}} \tilde{x} \\
& =\left[\begin{array}{c}
\mathbf{w}_{\mathrm{a}} \\
\mathbf{w}_{\mathrm{b}}
\end{array}\right]^{\mathrm{H}} \tilde{x} \\
& =\mathbf{w}_{\mathrm{a}}^{\mathrm{H}} \widetilde{\mathbf{x}}_{1}+\sum_{m=2}^{M} \mathbf{w}_{m}^{\mathrm{H}} \widetilde{\mathbf{x}}_{m},
\end{aligned}
$$

where $\mathbf{w}_{\mathrm{a}}=\left[\begin{array}{llll}w_{11} & w_{21} & \cdots & \tilde{w}_{N 1}\end{array}\right]^{\mathrm{H}}$ and $\mathbf{w}_{m}=$ $\left[\begin{array}{llll}w_{1 m} & w_{2 m} & \cdots & w_{N m}\end{array}\right]^{\mathrm{H}}$ are $N \times 1$ spatial and temporal weight vector, respectively, and the calculation method is shown in Section 2.2. The vector $y(k)$ contains three components consisting of signal, interference, and noise sources. The objective of proposed method is to minimize the following equation:

$$
\begin{aligned}
\left\langle\widetilde{\mathbf{M}}, \tilde{\mathbf{w}}_{\mathrm{a}}, \widetilde{\mathbf{w}}_{\mathrm{b}}\right\rangle=\min _{\mathbf{w}_{\mathrm{a}}, \mathbf{w}_{\mathrm{b}}} E\{ & \left.\|y(k)-d(k)\|^{2}\right\} \\
=\min _{\mathbf{w}_{\mathrm{a}}, \mathbf{w}_{\mathrm{b}}} E\{ & \| \mathbf{R}_{11}^{-1} \mathbf{a}_{\theta_{s}, \phi_{s}} \widetilde{\mathbf{x}}_{\mathrm{a}}-\mathbf{R}_{\mathrm{bb}}^{-1} \mathbf{d}_{\theta, \phi} \\
& \left.\times\left(\mathbf{d}_{\theta, \phi}^{\mathrm{H}} \mathbf{R}_{\mathrm{bb}}^{-1} \mathbf{d}_{\theta, \phi}\right)^{-1} \tilde{\mathbf{x}}_{\mathrm{b}}-d(k) \|^{2}\right\},
\end{aligned}
$$

where $d(k)$ is the ideal antenna array output. In practical applications, $\mathbf{R}_{11}$ and $\mathbf{R}_{\mathrm{bb}}$ are unattainable. Thus, the sample covariance matrices $\widetilde{\mathbf{R}}_{11}$ and $\widetilde{\mathbf{R}}_{\mathrm{bb}}$ are employed rather than $\mathbf{R}_{11}$ and $\mathbf{R}_{\mathrm{bb}}$. Equation (9) is obtained through iterative optimization method. Note that $\mathbf{a}_{\theta_{s}, \phi_{s}}$ and $\mathbf{d}_{\theta, \phi}$ are both vectors of desired signals but different only in size of dimension. The following is a step-by-step description of now to obtain the estimated $\tilde{\mathbf{w}}_{\mathrm{a}}$ and $\tilde{\mathbf{w}}_{\mathrm{b}}$ in order to obtain the estimated mutual coupling matrix $\widetilde{\mathbf{M}}$ iteratively.

Step 1. Use known desired array steering vector $\mathbf{a}_{\theta_{s}, \phi_{s}}$ to estimate the $\tilde{\mathbf{w}}_{\mathrm{a}}$, and considering the $\tilde{\mathbf{w}}_{\mathrm{a}}$ as initial spatial weight $\tilde{\mathbf{w}}_{\mathrm{a}}(0)$.

Step 2. Use the estimated $\widetilde{\mathbf{w}}_{\mathrm{a}}(M+1)((M+1)$ th iteration $)$ to construct $\tilde{\mathbf{w}}_{\mathrm{b}}$ at $M$ th iteration.

Step 3. Use (8) to estimate $\widetilde{\mathbf{M}}$.

Step 4. Use (7) to estimate $\widetilde{\mathbf{w}}_{\mathrm{b}}$ with the estimated $\widetilde{\mathbf{M}}$.

Step 5. If the algorithm converges, then stops. Otherwise, go to Step 2 to continue.

2.4. Performance Criterion. The performance measures are adopted to assess the performance of mutual coupling compensation and beamforming technique. $n$th antenna in 
(1) can be decomposed into three components: signal $z_{s}(k)$, interference $z_{r}(k)$, and noise $z_{v}(k)$ :

$$
x_{n}(k)=z_{s}(k)+z_{r}(k)+z_{v}(k) .
$$

Similarly, the vector $y(k)$ in (12) consists of three components: desired signal $y_{s}(k)$, interference $y_{r}(k)$, and noise $y_{v}(k)$ after the linear combination operation (12):

$$
y(k)=y_{s}(k)+y_{r}(k)+y_{v}(k) .
$$

The input signal-to-interference plus noise ratio (SINR) is considered as the ratio (in $\mathrm{dB}$ ) between the desired signal and other components, or

$$
\mathrm{SINR}_{\text {in }}=10 \log _{10}\left(\frac{\left\|z_{s}\right\|^{2}}{E\left\{\left\|z_{r}\right\|^{2}+\left\|z_{v}\right\|^{2}\right\}}\right) .
$$

The input interference-to-signal ratio (ISR) is defined as

$$
\text { ISR }=10 \log _{10}\left(\frac{\left\|z_{r}\right\|^{2}}{\left\|z_{s}\right\|^{2}}\right) .
$$

Use the superposition principle, and the SINR at the beamformer output can be evaluated as

$$
\operatorname{SINR}_{\text {out }}=10 \log _{10}\left(\frac{\left\|w^{\mathrm{H}} z_{s}\right\|^{2}}{E\left\{\left\|w^{\mathrm{H}} z_{r}\right\|^{2}+\left\|w^{\mathrm{H}} z_{v}\right\|^{2}\right\}}\right) .
$$

From (16) and (18), the improvement in SINR provided by the beamformer can be defined as

$$
\mathrm{SINR}_{\text {imp }}=\mathrm{SINR}_{\text {out }}-\mathrm{SINR}_{\text {in }} .
$$

This quality measure assesses the potential of the proposed algorithm to eliminate the power of interference in the incoming signal and meanwhile maintain the desired signal power. Besides SINR improvement factor, the signal acquisition margin (SAM) is utilized to determine the signal acquisition threshold [3]. It is a measure of the signal and noise peak values after correlation. Assume that $A_{s}$ is the peak value of the GPS signal in the acquisition diagram after correlation and $A_{v}$ is the peak value in correspondence to interference and noise. The SAM is defined as

$$
\mathrm{SAM}=10 \log _{10}\left(\frac{A_{s}}{E\left\{A_{v}\right\}}\right) .
$$

Thus, a high SAM indicates a successful rate in signal acquisition.

\section{Numerical Examples}

In this section, computer simulations are made to evaluate the performance of the proposed methods. The performance of proposed method and typical adaptive array technique $[8,16]$ is compared and evaluated. Three cases of simulations are presented for a $2 \times 2$ and $3 \times 3$ uniform rectangular array (URA). In the first scenario, the rectangular array operating in nominal mode is demonstrated. The second depicts a scenario where the interference is present and mutual coupling is absent. The third indicates the array processing under the condition of mutual coupling and interference. The antenna locations (in meters) in half-wavelength $(\lambda / 2)$ spacing with the array operate at IF $4.092 \mathrm{MHz}$. Each antenna has 5 taps delay and the direction of the desired signal is known a priori. Let the desired signal-to-noise ratio (SNR) in all simulations be $-20 \mathrm{~dB}$. The intensity of the received power of its immediate neighbors $\delta$ is between 0.1 and 0.5 .

The number of antenna elements $N$ is associated with the number of broadband interferences that can be cancelled by the spatial-temporal beamforming algorithm. In general, the number of broadband interferences that can be eliminated by the spatial-temporal filtering corresponds to $N-1$. Three scenarios with the main parameters illustrated in Table 1 are simulated. One desired signal at broadside $\left(\theta=40^{\circ}, \phi=\right.$ $120^{\circ}$ ) and two directional broadband interference sources at $\left[\left(\theta=40^{\circ}, \phi=15^{\circ}\right),\left(\theta=40^{\circ}, \phi=170^{\circ}\right)\right]$ are generated in the simulations where the desired signal and each of the interference signals are uncorrelated. 100 Monte Carlo simulations are conducted and the length of the available data record is $40 \mathrm{~ms}$.

Figure $1(a)$ illustrates the use of URA $(2 \times 2)$ array structure under ISR as $50 \mathrm{~dB}$ and shows the result of gain pattern with/without the proposed algorithm. The gain pattern can be illustrated when the spatial-temporal weight of proposed method satisfies (13). The figure demonstrates that under the effect of mutual coupling, the interference cannot be fully mitigated due to the decrease of gain in the DoA of desired signal and wrong direction of interference mitigation. Through mutual coupling compensation, the gain of interference direction can be effectively mitigated and the error between desired direction and simulated direction is eliminated. On the other hand, the gain of desired direction is enhanced. Figure 1(b) shows similar results in the use of URA $(3 \times 3)$ array structure, but only different in the increase of space freedom regarding interference mitig- ation in comparison with simulation results of URA $(2 \times 2)$ structure. Figure 1 demonstrates that Sarkar's method can maintain the direction gain of desired signal under mutual coupling effect. On the contrary, the mitigation of direction gain regarding interference signal is not effective. Figure 2 describes the plot of SINR normalized to an asymptotic solution versus number of samples for $3 \times 3$ URA. In these arrays, the estimates of the improvement in SINR are assessed as a function of the ISR and plotted in Figure 3. The figure illustrates that the more the increase of ISR, the more the improvement of SINR after decoupling and interference mitigation. This is more obvious under $3 \times 3$ URA structure. The conjugate gradient method is demonstrated in literature review [8].

\section{Test Hardware and Experiment Results}

This session depicts how embedded system is implemented in proposed method with regards to mutual coupling compensation and interference mitigation and meanwhile presents how to construct an embedded system as a platform combination antenna array modules to receive GPS signal. 


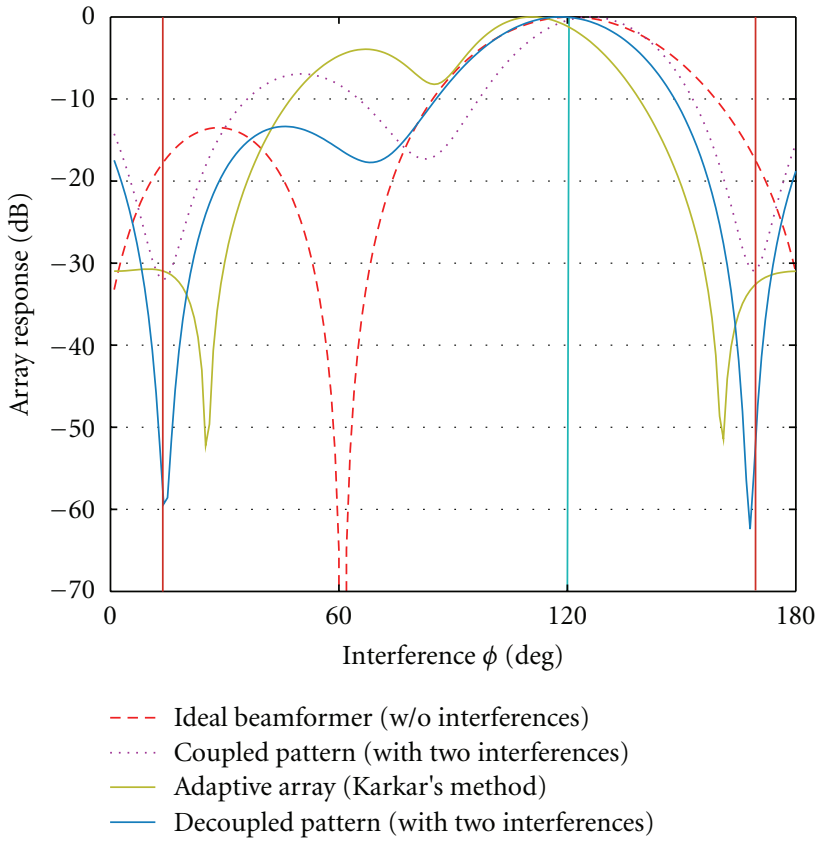

(a) URA $(2 \times 2)$

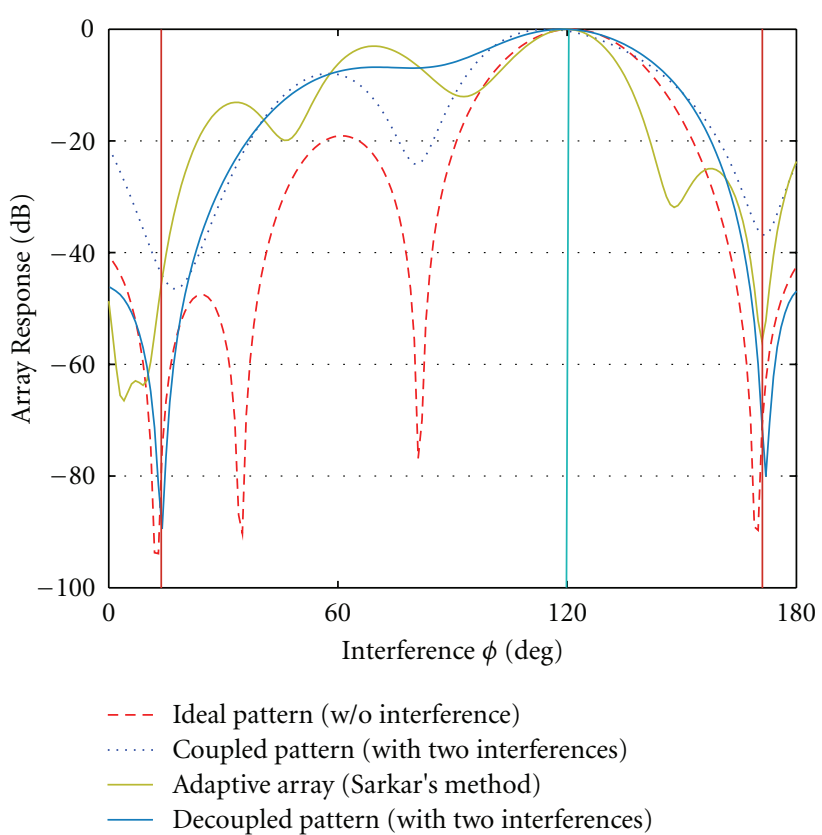

(b) URA $(3 \times 3)$

FIGURE 1: The beam pattern in different scenarios for ISR $=50 \mathrm{~dB}$

TABLE 1: Parameters setup of test scenarios.

\begin{tabular}{|c|c|c|c|c|c|}
\hline & $\begin{array}{c}\text { Number of antenna } \\
\text { element (URA) }\end{array}$ & $\begin{array}{l}\text { Mutual coupling exist? } \\
\text { (Yes/No) }\end{array}$ & ISR & $\begin{array}{l}\text { Interference } \\
\text { direction }\left(\theta_{j}, \phi_{j}\right)\end{array}$ & $\begin{array}{l}\text { Desired GPS signal } \\
\text { direction }\left(\theta_{s}, \phi_{s}\right)\end{array}$ \\
\hline \multirow{2}{*}{ Scenario 1} & $4(2 \times 2)$ & \multirow{2}{*}{ No } & \multirow{2}{*}{ None } & \multirow{2}{*}{ None } & \multirow{2}{*}{$\left(40^{\circ}, 120^{\circ}\right)$} \\
\hline & $9(3 \times 3)$ & & & & \\
\hline \multirow{2}{*}{ Scenario 2} & $4(2 \times 2)$ & No & \multirow{2}{*}{$10 \sim 80 \mathrm{~dB}$} & $\left(40^{\circ}, 15^{\circ}\right)$ & \multirow{2}{*}{$\left(40^{\circ}, 120^{\circ}\right)$} \\
\hline & $9(3 \times 3)$ & No & & $\left(40^{\circ}, 170^{\circ}\right)$ & \\
\hline \multirow{2}{*}{ Scenario 3} & $4(2 \times 2)$ & Yes & \multirow{2}{*}{$10 \sim 80 \mathrm{~dB}$} & $\left(40^{\circ}, 15^{\circ}\right)$ & \multirow{2}{*}{$\left(40^{\circ}, 120^{\circ}\right)$} \\
\hline & $9(3 \times 3)$ & Yes & & $\left(40^{\circ}, 170^{\circ}\right)$ & \\
\hline
\end{tabular}

TABLE 2: Corresponding output signal.

\begin{tabular}{lcc}
\hline SGN & MAG & Value \\
\hline 0 & 1 & +3 \\
0 & 0 & +1 \\
1 & 0 & -1 \\
1 & 1 & -3 \\
\hline
\end{tabular}

4.1. Hardware Description. The experiment structure includes an antenna array platform and embedded system to implement the proposed algorithm. The hardware structure is illustrated in Figure 4. Four antennas make up the antenna array platform in rectangular shape with the spacing between each antenna as L1 half-wave length $(9.5 \mathrm{~cm})$. Each antenna is, respectively, connected to four RF front-end modules and the external oscillator and buffer can provide clock signal for each module to simultaneously receive GPS signals. GPS signal is converted to 2-bit digital intermediate frequency (IF) for output through the analog-to-digital converter
TABLE 3: Experiment results for PRN 11(SAM detection threshold $=5 \mathrm{~dB}$ ).

\begin{tabular}{lcccccc}
\hline \multirow{2}{*}{ SAM } & \multicolumn{3}{c}{$\begin{array}{l}\text { Without self-tuning } \\
\text { synthesis algorithm }\end{array}$} & \multicolumn{3}{c}{$\begin{array}{l}\text { With self-tuning } \\
\text { synthesis algorithm }\end{array}$} \\
\hline ISR & $\lambda$ & $\lambda / 2$ & $\lambda / 4$ & $\lambda$ & $\lambda / 2$ & $\lambda / 4$ \\
\hline 10 & 7.12 & 7.83 & 5.3 & 7.23 & 7.62 & 6.28 \\
20 & 5.81 & 6.44 & - & 7.19 & 7.44 & 6.32 \\
30 & - & 5.11 & - & 6.99 & 7.23 & 5.21 \\
40 & - & - & - & 6.95 & 7.19 & - \\
50 & - & - & - & 6.91 & 7.15 & - \\
\hline
\end{tabular}

(ADC) in each module. The output signal is sent to the development board of embedded system to conduct mutual coupling compensation and interference mitigation and the proposed method can be implemented through embedded system development kit. The computer then verifies and analyzes whether the received signal can efficiently mitigate interference and compensate mutual coupling through 


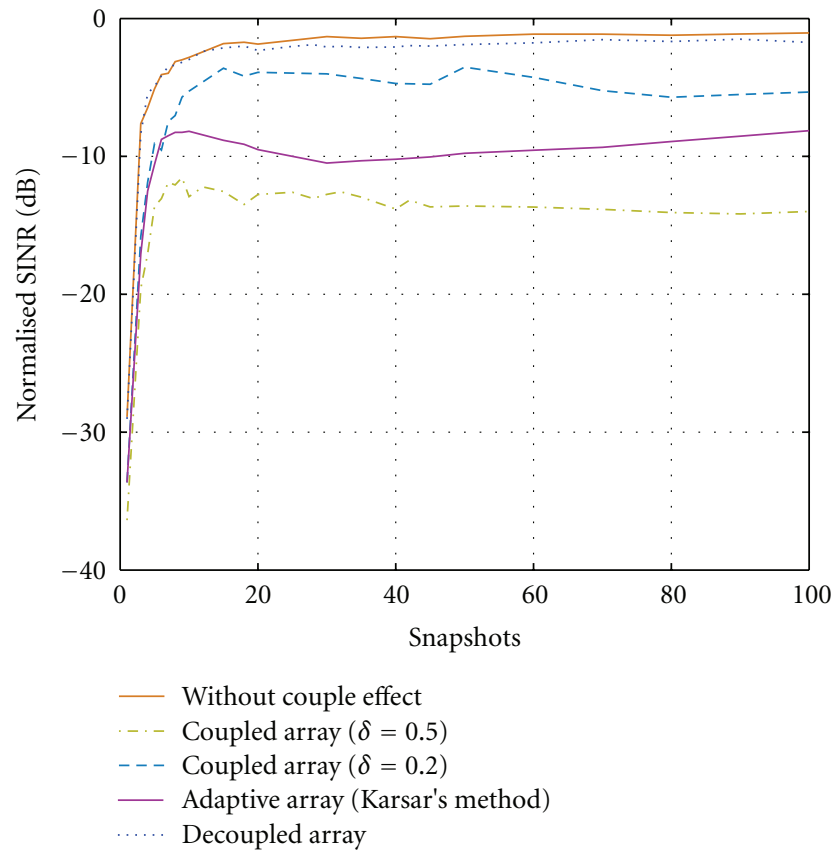

FIGURE 2: Normalized SINR in the array output before and after decoupling (URA $3 \times 3$ ).

TABLE 4: C/No results with/without proposed method.

\begin{tabular}{|c|c|c|c|c|c|}
\hline \multirow[b]{2}{*}{ PRN } & \multicolumn{4}{|c|}{ Without proposed algorithm } & \multirow{2}{*}{$\begin{array}{l}\text { With } \\
\text { proposed } \\
\text { algorithm }\end{array}$} \\
\hline & $\mathrm{A} 1$ & A2 & $\begin{array}{c}\mathrm{A} 3 \\
\mathrm{C} / \mathrm{No}(\mathrm{dB}-\mathrm{Hz})\end{array}$ & A4 & \\
\hline 4 & 38.41 & 38.30 & 36.62 & 38.31 & 38.81 \\
\hline 8 & 37.22 & 34.17 & 35.44 & 36.45 & 38.46 \\
\hline 11 & 32.24 & 35.34 & 34.71 & 32.75 & 39.05 \\
\hline 17 & - & - & 33.43 & - & 36.87 \\
\hline 20 & 36.28 & 37.14 & 36.64 & 35.75 & 38.52 \\
\hline 25 & 32.76 & 36.82 & 37.70 & 38.11 & 39.30 \\
\hline 27 & - & 35.22 & - & 35.23 & 39.56 \\
\hline 28 & 36.80 & 36.49 & - & - & 37.67 \\
\hline 19 & 36.88 & 37.31 & - & 34.11 & 38.26 \\
\hline
\end{tabular}

embedded system to provide acquisition and tracking for satellite positioning. The function of each component in the system structure is demonstrated as follows.

4.1.1. GPS Antenna. The experiment adopts four MK-76 GPS antennas [17], with each antenna gain as $26 \mathrm{~dB}$ and noise figure as $2.0 \mathrm{~dB}$.

4.1.2. RF Front-End. The RF front-end module consists of RF front-end IC produced by merged Nemerix Inc., RF low noise amplifier (LNA), mixer and 2-bit ADC module. The ADC output is "SIGN" and "MAG" bit data output which has to be transferred in order to correspond to suitable voltage level in embedded development board. The corresponding relation is illustrated in Table 2. The experiment requires at least four RF front-end modules with the sampling frequency as $16.368 \mathrm{MHz}$ and digital IF as $4.092 \mathrm{MHz}$.

4.1.3. Digital Signal Processing Development Kit (DSP DEVKIT-2S60). The DSP Development Kit, Stratix II Edition, offers engineers with a complete system-on-aprogrammable-chip (SoPC) solution. The kit is developed and designed by Altera company, which adopts highperformance FPGA DSP application and structured application specific integrated circuit (ASIC) platform. The complete Altera DSP Development Kit includes DSP development board and Quartus II 6.0, SoPC Builder 6.0, and NiosII 6.0 in software. The major components in development board consist of Nios II core processor, on-chip memory, serial peripheral interface (SPI), universal asynchronous receiver/transmitter (UART), direct memory access (DMA) controller, parallel input/output (I/O) interface, avalon bus, and Timer, which are integrated in an Altera's Stratix II FPGA device.

The Stratix II EP2S60 DSP development board is used in this paper and this board provides a hardware platform that designers can utilize to start developing DSP systems according to Stratix II devices. Combined with DSP intellectual property (IP) from Altera and Altera megafunction partners program $\left(\mathrm{AMPP}^{\mathrm{SM}}\right)$ partners, users can quickly develop powerful DSP systems. The development board includes some memories and hardware transmission interface, such as $32 \mathrm{MB}$ synchronous dynamic random access memory (SDRAM) and $1 \mathrm{MB}$ static random access memory (SRAM), joint test action group (JTAG), and RS232, which employs SoPC Builder to configure the system on a chip and also generates the Avalon switch fabric to connect all ports. More detailed information can be found in [18].

4.2. Experiment System Establishment. With respect to embedded system, the inbuilt Megafunction in Quartus II [19] is employed to design spatial-temporal structure and the inbuilt design tool SoPC builder in Quartus II is adopted to construct Nios II CPU [20] for communication with peripheral interface. The self-tuning synthesis filter algorithm adopts C-language to write and implement in Nios II integrated device electronics (IDE) [21]. The proposed algorithm is to adjust weight, estimate mutual coupling matrix, and have the value sent to the designed spatialtemporal structure in Quartus II to allow mutual coupling compensation towards GPS signal and mitigation towards noise, interference.

The following context will describe how to apply embedded development board to implement the proposed algorithm and conduct signal acquisition analysis of the processed signal using Matlab software.

Step 1. Four RF front-end modules are connected to four antennas, respectively, on experiment platform. Then, the self-designed circuit interface board is inserted to I/O interface of embedded development board. Then, the Quartus II software is utilized to construct transmission interface 


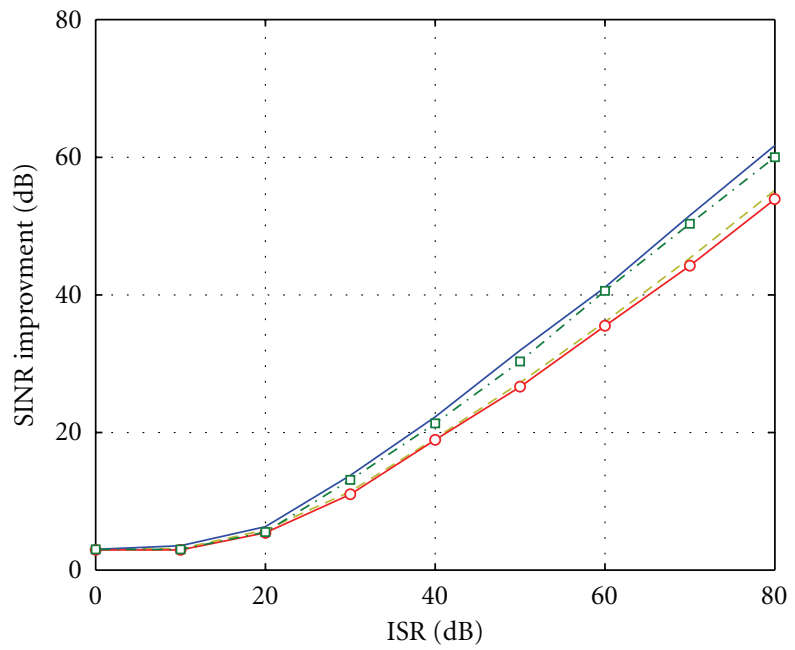

- Without couple effect
$\square-$ Coupled array
-- Adaptive array (Karsar's method)
$\square-$ Decoupled array (proposed)

(a) URA $2 \times 2$

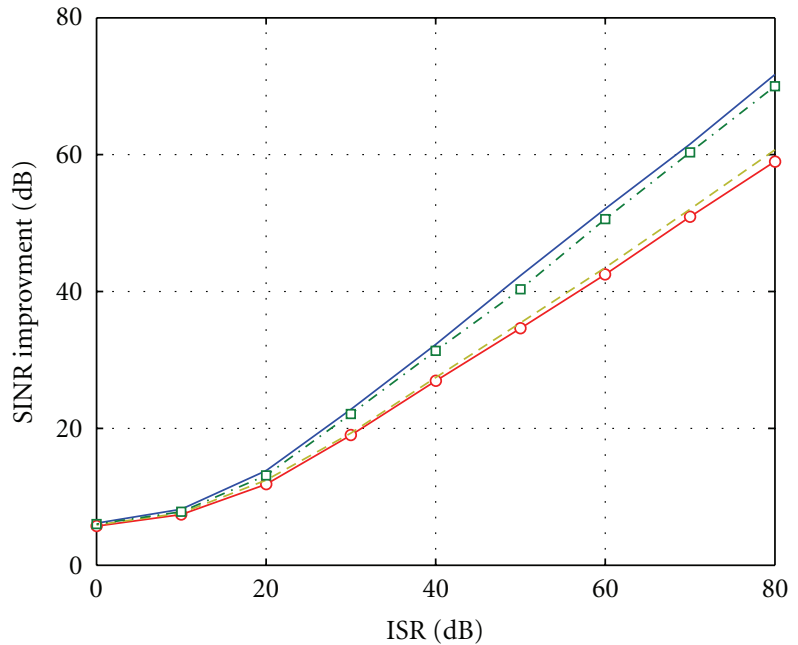

— Without couple effect
- - Coupled array
$-\ldots$ Adaptive array (Sarkar' method)
- - Decoupled array (proposed)

(b) URA $3 \times 3$

FIgURE 3: SINR improvement for $2 \times 2$ and $3 \times 3$ URA structure.

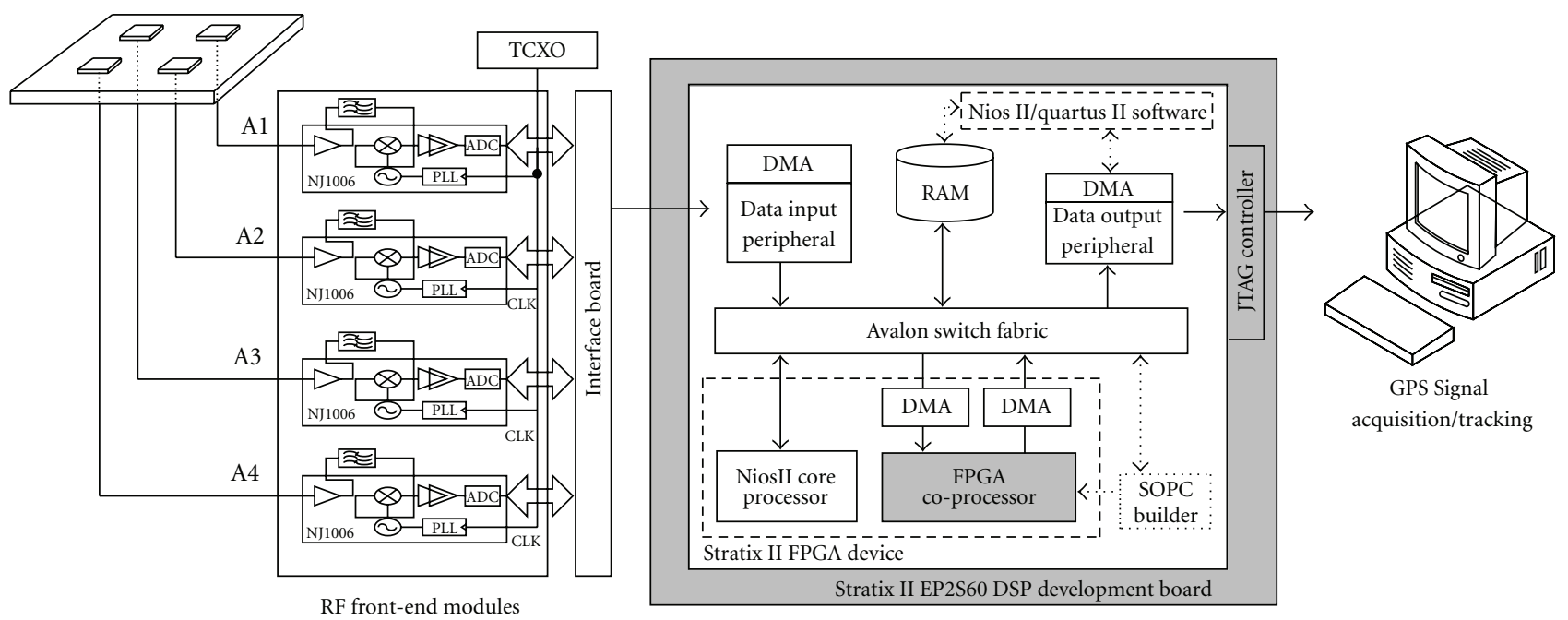

Figure 4: Antenna array experiment system.

between RF front-end module and embedded board. The goal is to allow embedded board to receive GPS signals from RF front-end modules. First, the pin name of signal input is set and its location is assigned to the point of expansion Interface in DSP development board. Then, the "altpll" megafunction is added in the top-level Quartus II design to create a phase-locked loop (PLL) clock output. This process constitutes the transmission interface. Each RF front-end module outputs 2-bit digital signal. Thus, an antenna array composed of four antennas makes 8-bit output. The transmission interface is to test whether the embedded system can accurately receive GPS data without self-tuning algorithm.
The Quartus II software is adopted to construct spatialtemporal filter where each antenna is composed of 5-tap finite impulse response (FIR) filter. Hence, four antenna sets make up 20 taps. Figure 5 demonstrates the structure of self-tuning filter. RF front-end modules transfer incoming signal to IF digital signal, which is sent to embedded development board for spatial-temporal signal processing. The input data has to multiply the weight of real and image, respectively, after each delay time, the result of which is summed altogether for output.

Step 2. The spatial-temporal module constructed in Quartus II software is incorporated to user logic program and 


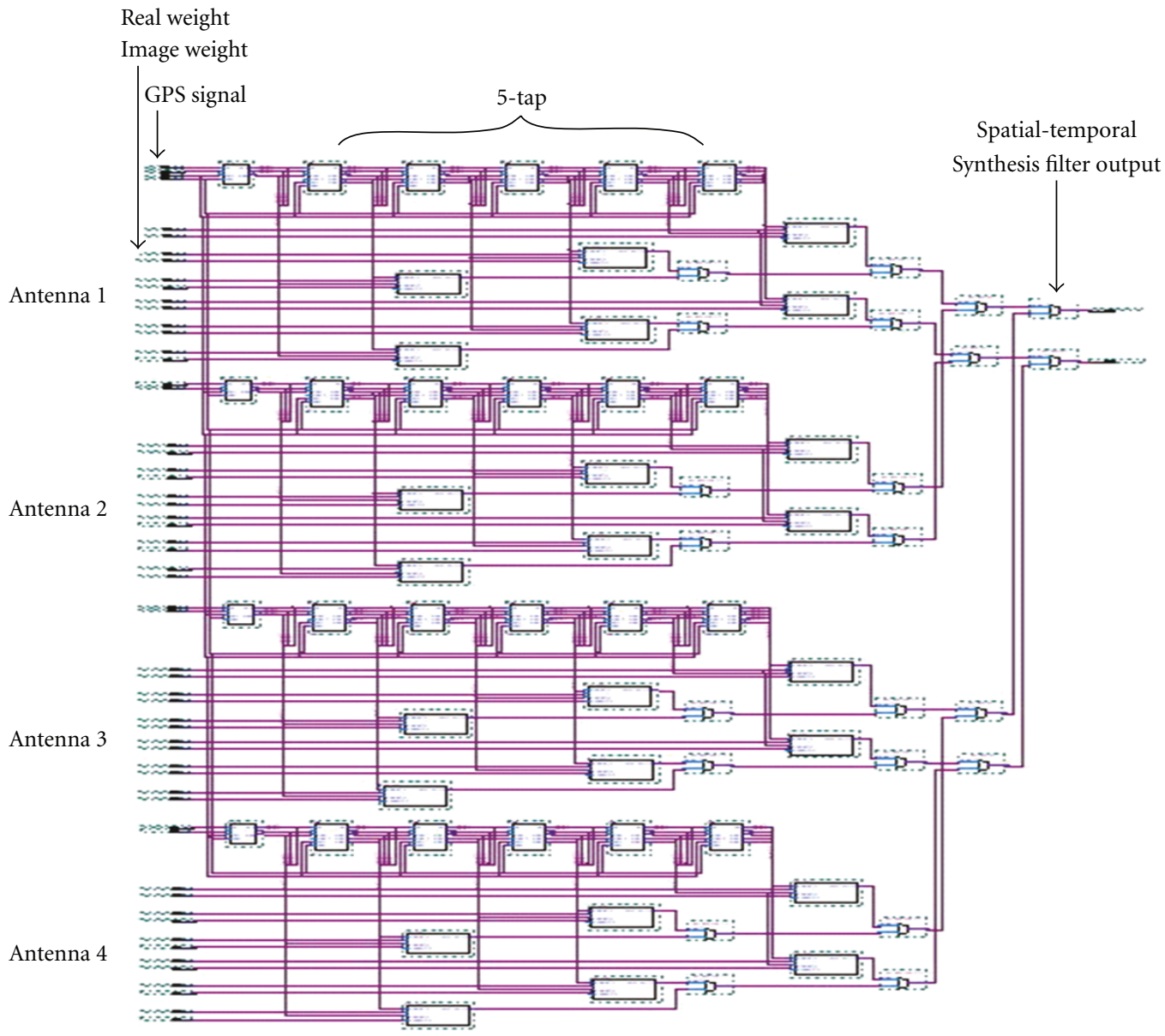

Figure 5: Self-tuning synthesis filter structure.

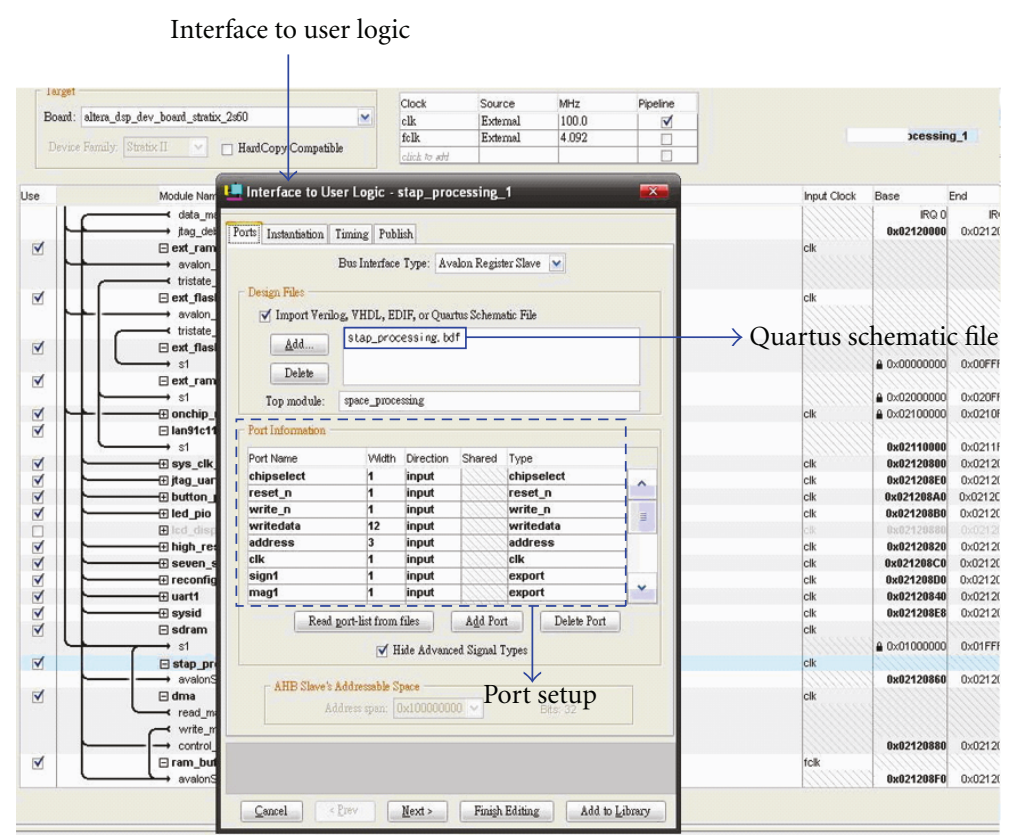

FIGURE 6: SoPC builder interface. 


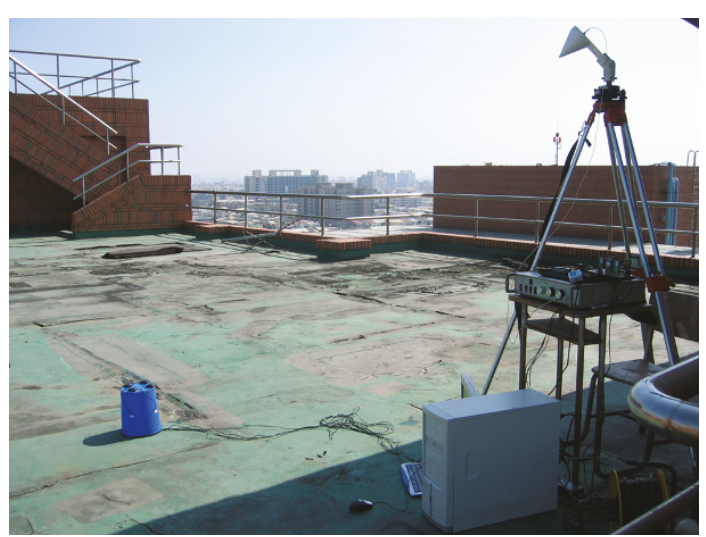

FIGURE 7: Photo of experiment location.

connected to Avalon. The accomplishment in setting up related parameter can yield CPU core designed by user (as shown in Figure 6). The translation starts to execute upon the incorporation of designed Nios CPU to the constructed spatial-temporal structure in Quartus II and the joint connection between them. If no error occurs in the translation, the * .sof file generates and is written to FPGA board.

Step 3. Select a spacious ground and employ the designed platform to receive GPS signal. The length of each data reception is about $3 \mathrm{~ms}$. Have the received data sent to spatial-temporal processing module in Quartus II for signal processing. Through the operation of dynamic memory access (DMA), store the $3 \mathrm{~ms}$ received GPS signal to external SDRAM for Nios II to execute adaptive algorithm.

SoPC Builder database consists of a processor and a large amount of IP core. This system adopts Nios II processor, DMA, SDRAM, user logic, JTAG, and UART and has the constituted spatial-temporal filter modules in Quartus II incorporated into "User logic", which is connected to Avalon shown in Figure 6. The designed CPU is generated as the transmission interface between Quartus II and Nios II. The calculated weights will be delivered to spatial-temporal module in Quartus II through Nios II CPU by way of iteration. After several iterations, Quartus II performs to acquire the output data of spatial-temporal processing.

Step 4. Conduct signal acquisition analysis of the processed data using Matlab software.

4.3. Results. The location of experiment test is selected at longitude $22.9^{\circ}$ and latitude $120.2^{\circ}$, shown in Figure 7 . The observable GPS satellites are depicted in the sky plot of Figure 8. Eight GPS satellites are observed. The location of the interferer is at azimuth $312^{\circ}$ and elevation $53^{\circ}$ and ISR is about $30 \mathrm{~dB}$. The initial location of each satellite can be obtained by YUMA data [22]. The data is stored in randomaccess memory (RAM) on-chip in development board, and the initial weight value is also stored in the RAM onchip. In the experiment, the embedded development board is switched on to receive GPS signals and then conducts

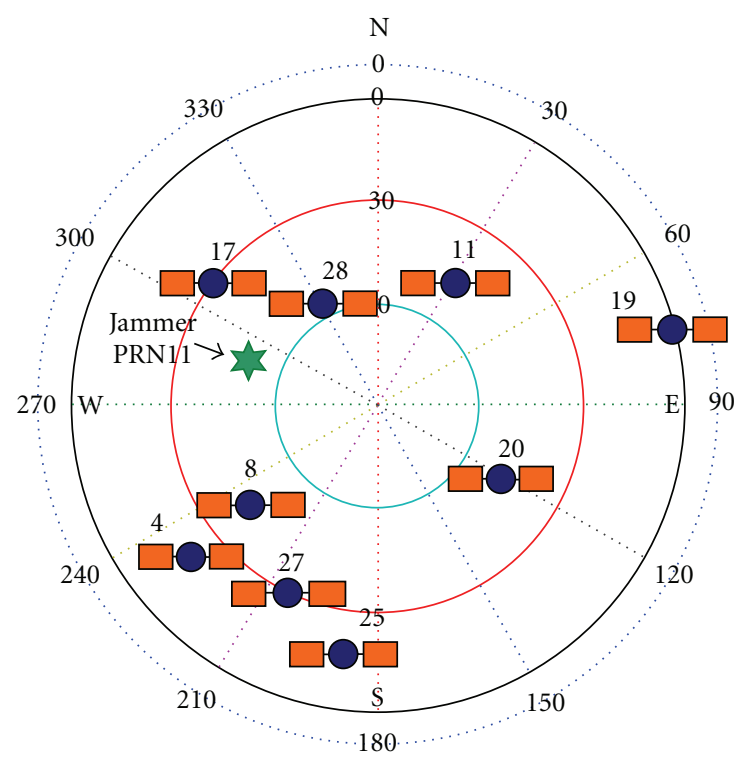

FIGURE 8: Sky plot of GPS satellites and near-field pseudolite interference.

iteration process through proposed algorithm when the weight value converges. The output data is postprocessed by the Matlab software for signal acquisition. The nominal SAM with regards to each satellite is computed. The cochannel interference is then switched on and the data consisting of components such as GPS satellites, the interference, and noises is processed by the proposed algorithm. Figure 9 shows the digital waveform from four RF front-end modules in system PLL. The figure illustrates that the GPS signal from each antenna is successfully received by embedded development board. Table 3 describes the signal acquisition result of PRN 11 with/without the proposed algorithm under different antenna spacing with mutual coupling matrix known a priori. The table illustrates that the use of proposed algorithm can lower the impact of decreased acquisition performance due to the antenna spacing less than $\lambda / 2$. Figure 10 shows the signal acquisition result of each antenna (the antenna spacing is $\lambda$ ) regarding PRN 11 without proposed method and it indicates the coexistence of nearfield pseudolite interference (ISR $=30 \mathrm{~dB}$ ) and true PRN 11 satellite signal. The figure presents that signal acquisition process locks cochannel interference signals, which leads to error in signal tracking and increase in positioning error. The acquisition result of antenna 2 (A2) differs due to unsimultaneous reception time of each antenna, incoherent antenna characteristics, or phase error caused by mutual coupling. Nevertheless, it is for certain that the acquisition magnitude of interference signal is far stronger than that of live satellite signal (PRN 11).

Figure 11 describes that when the weight value of spatial-temporal filter converges after iteration of proposed algorithm, the acquisition magnitude of interference signal is weak. This is because the direction gain of interference has been nulled. Figure 12 shows gain pattern after beamforming and it indicates that the proposed algorithm can efficiently 


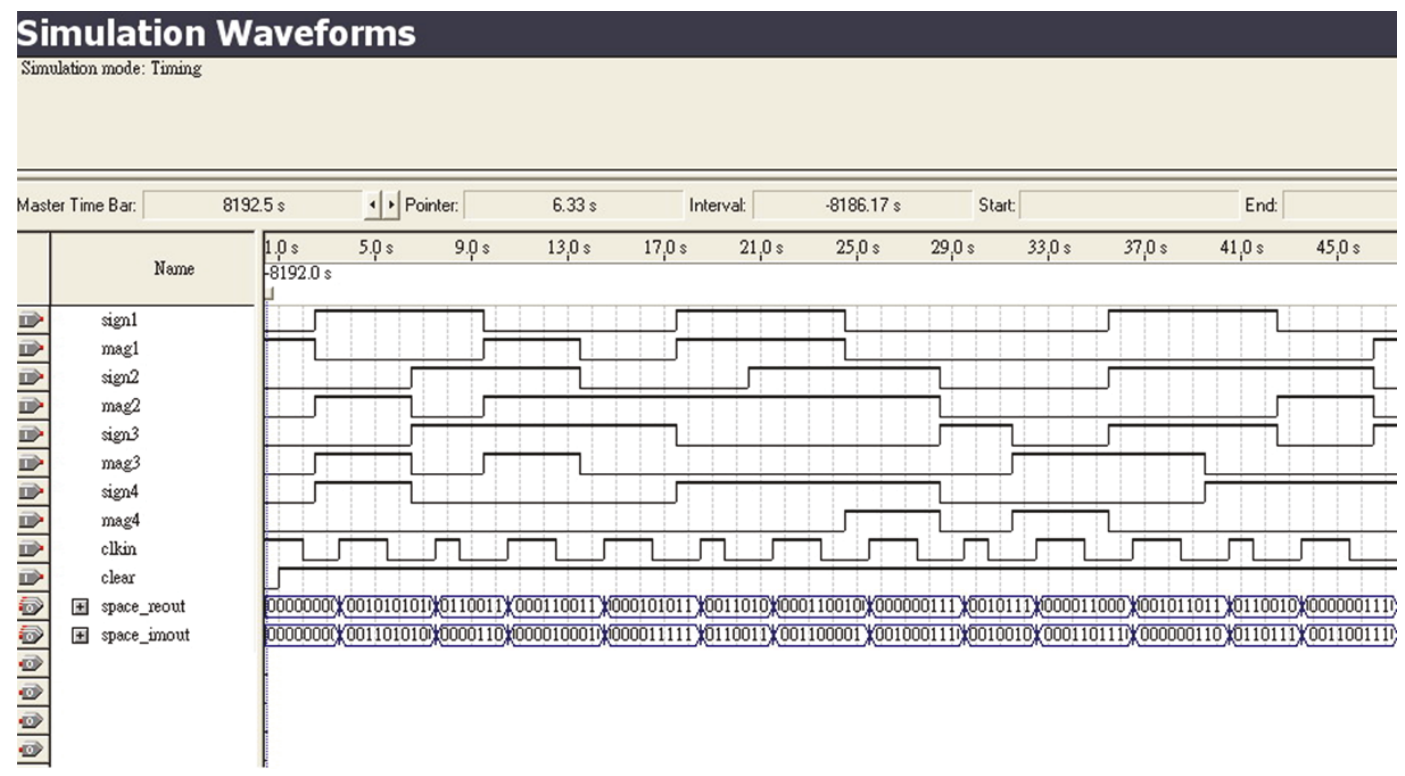

FIgURE 9: The waveforms of hardware output.

Code phase $=423.1875 \quad$ Doppler shift $=4000 \mathrm{~Hz}$

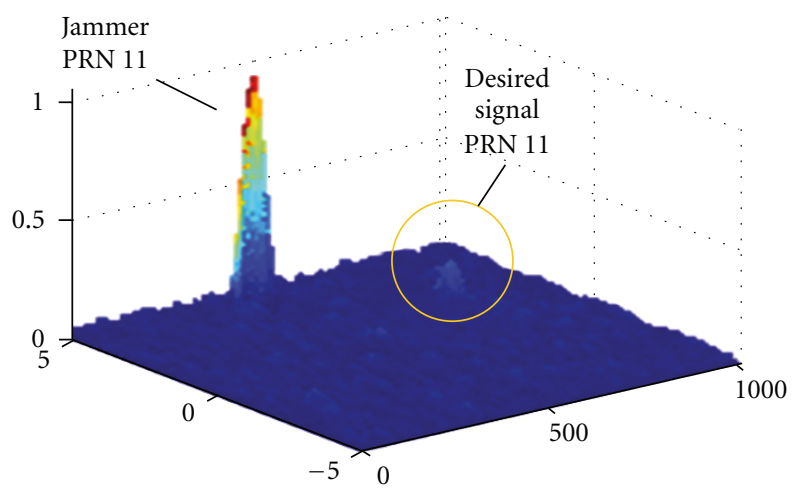

(a)

Code phase $=423.125$ Doppler shift $=4000 \mathrm{~Hz}$

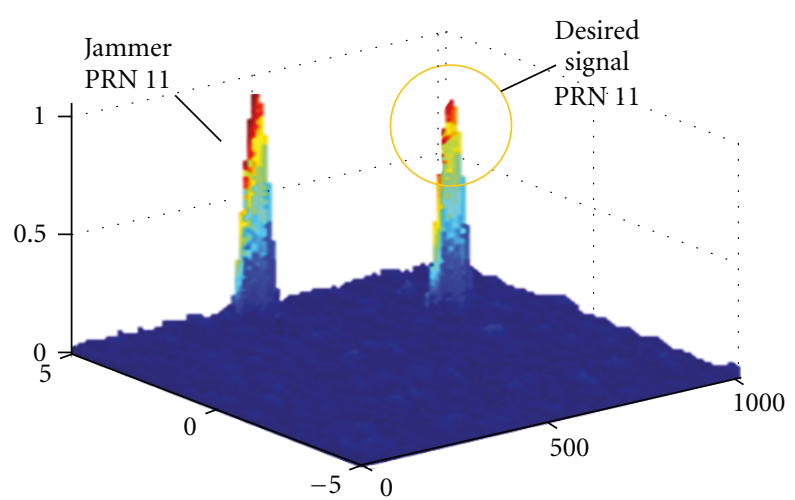

(c)
Code phase $=423.1875 \quad$ Doppler shift $=4000 \mathrm{~Hz}$

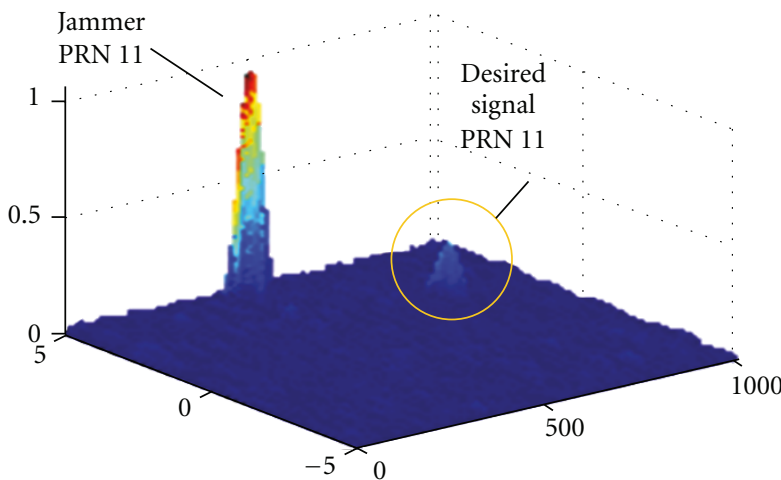

(b)

Code phase $=423.1875 \quad$ Doppler shift $=4000 \mathrm{~Hz}$

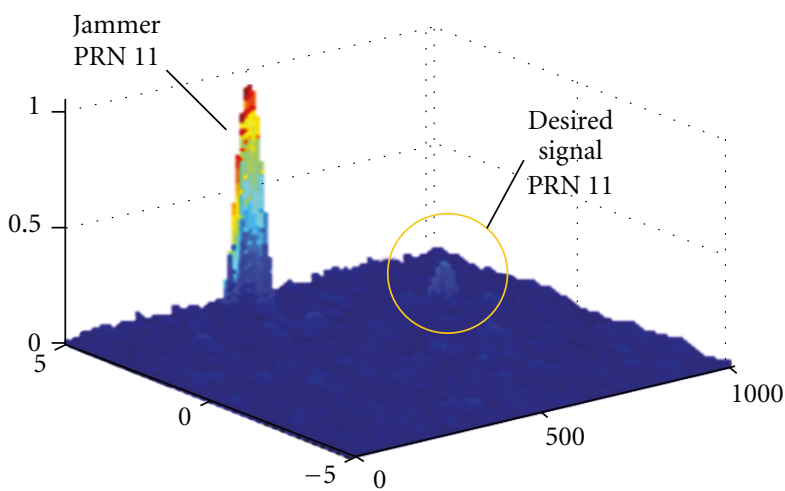

(d)

Figure 10: Acquisition result for antenna 1 (a), antenna 2 (b), antenna 3 (c), and antenna 4 (d). 


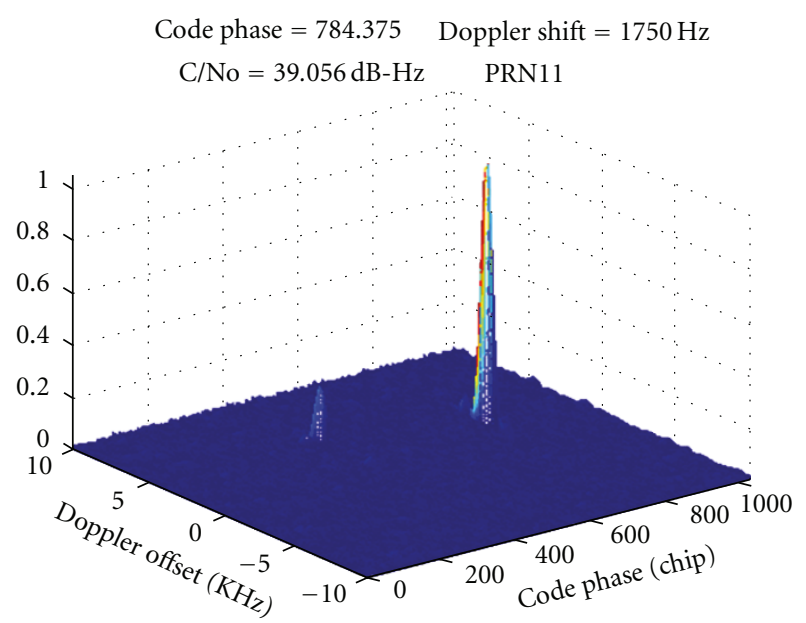

FIGURE 11: Acquisition result for PRN 11 using proposed algorithm.

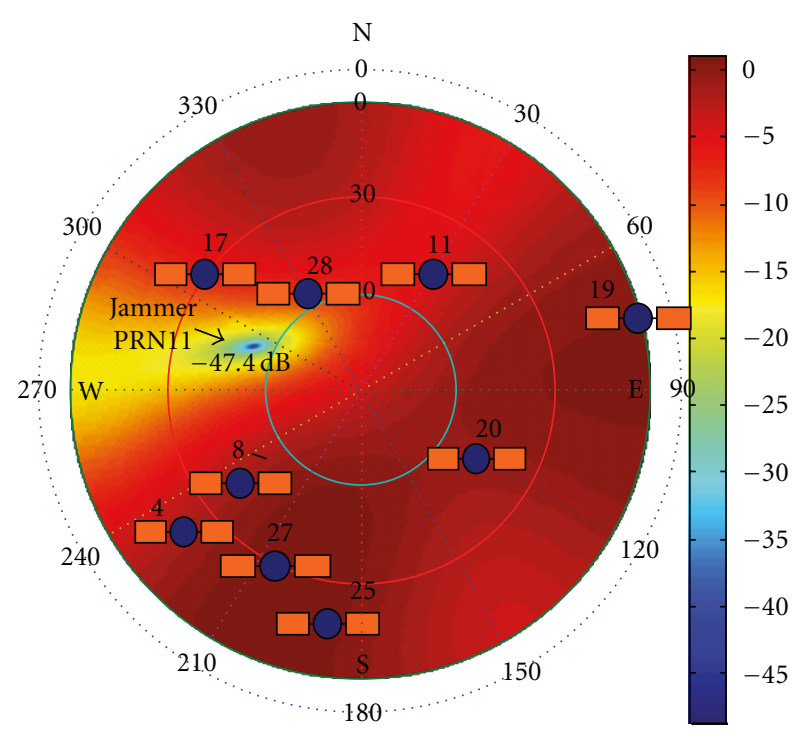

FIGURE 12: Gain pattern of antenna array.

mitigate cochannel interference (jammer PRN 11). The figure demonstrates that the direction gain of interference has been nulled to $-47.4 \mathrm{~dB}$. The direction gain of desired signals such as PRN 11, 28, and 17 is somewhat influenced (about $-4 \sim-11 \mathrm{~dB})$ whereas that of other satellites is between $0 \sim$ $-6 \mathrm{~dB}$. Through the direction gain of PRN 11, 28, and 17 is somewhat influenced; their carrier-to-noise ratio $(\mathrm{C} / \mathrm{No})$ values calculated as $39.05,37.67$, and 36.87 and shown in Table 3 are sufficient to provide signal tracking. In Table 4 the use of self-tuning synthesis algorithm does indeed enhance the $\mathrm{C} / \mathrm{No}$ of each satellite.

\section{Conclusion}

This paper proposes a self-tuning synthesis algorithm to simultaneously compensate for mutual coupling effect and mitigate interference. Simulation and experiment results are demonstrated to verify the feasibility of proposed method. In simulation, $2 \times 2$ and $3 \times 3$ URA structures are adopted to analyze the performance of proposed algorithm, respectively. The simulation result indicates that $2 \times 2$ and $3 \times 3$ URA can effectively compensate for mutual coupling effect (the maximum tolerable value is $\delta=0.5)$. With respect to interference mitigation for $2 \times 2$ URA, the SINR improvement reaches $5.5 \sim 60.2 \mathrm{~dB}$ with ISR between $20 \sim 80 \mathrm{~dB}$. Regarding $3 \times 3$ URA, the SINR improvement reaches $13.2 \sim$ $70.1 \mathrm{~dB}$. The use of $3 \times 3$ URA structure outperforms that of $2 \times 2$ URA structure. In addition, the more the increase of ISR, the more the improvement of SINR after decoupling process. This paper also constructs an SoPC-embedded experiment platform to verify the proposed method. As for the SoPC development system utilized in this paper, the design of point-to-point connection between logic gates can be adopted in Quartus II hardware. Thus, it saves the trouble of writing hardware language and the graphic user interface (GUI) is more accessible to users. Besides, the adoption of Nios II IDE in software is compatible to C-language, which makes it easier to write algorithm.

However, in consideration of cost, this paper utilizes $2 \times 2$ structure to analyze the performance of proposed algorithm and employ an embedded system to develop this algorithm in experiment. Due to the limitation in hardware, the use of only five delay taps leads to the confinement of performance in interference mitigation to $30 \mathrm{~dB}$. The experiment also analyzes the effect of different antenna spacing on the performance of proposed algorithm. The results reveal that the proposed method can efficiently compensate for mutual coupling caused by narrower antenna spacing even if it is $\lambda / 4$.

\section{Acknowledgments}

The author would like to thank the mechatronics laboratory, National Cheng Kung University to provide the instruments to conduct experiment and National Science Council in Taiwan to support this research under Grant NSC 98-2218E-020-003.

\section{References}

[1] J. J. Spilker and F. D. Natali, "Interference effects and mitigation techniques," in Global Positioning System: Theory and Applications, B. W. Parkinson and J. J. Spilker, Eds., American Institute of Aeronautics and Astronautics, Washington, DC, USA, 1996.

[2] C. L. Chang and J. C. Juang, "Performance analysis of narrowband interference mitigation and near-far resistance scheme for GNSS receivers," Signal Processing, vol. 90, no. 9, pp. 2676-2685, 2010.

[3] J.-C. Juang and C.-L. Chang, "Performance analysis of GPS pseudolite interference mitigation using adaptive spatial beamforming," in Proceedings of the Annual Meeting of the Institute of Navigation, pp. 1179-1187, Cambridge, Mass, USA, June 2005.

[4] D. H. Johnson and D. E. Dudgeon, Array Signal Processing: Concepts and Techniques, Prentice Hall, Upper Saddle River, NJ, USA, 1993. 
[5] S.-J. Kim and R. A. Iltis, "STAP for GPS receiver synchronization," IEEE Transactions on Aerospace and Electronic Systems, vol. 40, no. 1, pp. 132-144, 2004.

[6] B. Allen and M. Ghavami, Adaptive Array Systems: Fundamentals and Applications, John Wiley and Sons, New York, NY, USA, 2005.

[7] C.-L. Chang and J.-C. Juang, "Effect of array configurations on the performance of GNSS interference suppression," International Journal of Control, Automation and Systems, vol. 6, no. 6, pp. 884-893, 2008.

[8] T. K. Sarkar and N. Sangruji, "Adaptive nulling system for a narrow-band signal with a look-direction constraint utilizing the conjugate gradient method," IEEE Transactions on Antennas and Propagation, vol. 37, no. 7, pp. 940-944, 1989.

[9] I. J. Gupta and A. A. Ksienski, "Effect of mutual coupling on performance of adaptive arrays," IEEE Transactions on Antennas and Propagation, vol. 32, no. 5, pp. 785-791, 1983.

[10] E. M. Friel and K. M. Pasala, "Effects of mutual coupling on the performance of STAP antenna arrays," IEEE Transactions on Aerospace and Electronic Systems, vol. 36, no. 2, pp. 518527,2000 .

[11] T. Svantesson, "Modeling and estimation of mutual coupling in a uniform linear array of dipoles," in Proceedings of the IEEE International Conference on Acoustics, Speech, and Signal Processing (ICASSP '99), pp. 2961-2964, Phoenix, Ariz, USA, March 1999.

[12] H. Steyskal and J. S. Herd, "Mutual coupling compensation in small array antennas," IEEE Transactions on Antennas and Propagation, vol. 38, no. 12, pp. 1971-1975, 1990.

[13] F. Sellone and A. Serra, "A novel online mutual coupling compensation algorithm for uniform and linear arrays," IEEE Transactions on Signal Processing, vol. 55, no. 2, pp. 560-573, 2007.

[14] Z. Huang, C. A. Balanis, and C. R. Birtcher, "Mutual coupling compensation in UCAs: Simulations and experiment," IEEE Transactions on Antennas and Propagation, vol. 54, no. 11, pp. 3082-3086, 2006.

[15] C. L. Chang and J. C. Juang, "A new pre-processing approach against array uncertainty for GNSS," in Proceedings of the IEEE/ION PLANS, pp. 892-897, Monterey, Calif, USA, May 2008.

[16] R. S. Adve and T. K. Sarkar, "Compensation for the effects of mutual coupling on direct data domain adaptive algorithms," IEEE Transactions on Antennas and Propagation, vol. 48, no. 1, pp. 86-94, 2000.

[17] Sanav. San Jose Technology, Inc., http://www.sanav.com/.

[18] Altera Corporation, "Nios development board Stratix II edition ref. manual," 2006.

[19] Altera Corporation, "Quartus II development software ver. $5.1, " 2004$.

[20] Altera Corporation, Nios II Processor Reference's Handbook, 2004.

[21] Altera Corporation, Nios II Software Developer's Handbook, 2004.

[22] Navigation Center, http://www.navcen.uscg.gov/?pageName= gpsAlmanacs. 